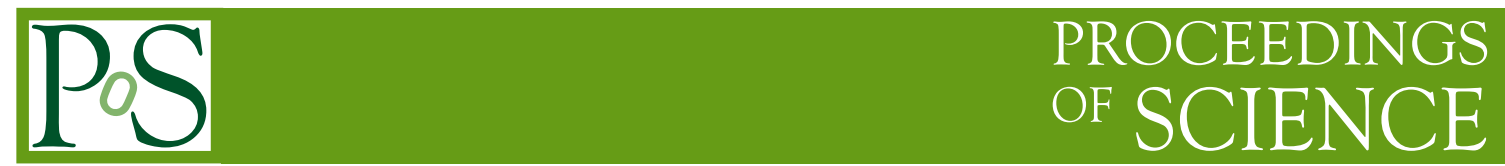

\title{
Structure and polarization of the inner jets
}

\author{
Mark Birkinshaw* \\ HH Wills Physics Laboratory, University of Bristol \\ E-mail: Mark.Birkinshaw@bristol.ac.uk
}

Polarization is an important indicator of the structure and composition of the jets in active galaxies. This article presents a brief review of the features of the polarization of jets and how those features are interpreted.

AGN Physics in the CTA Era-AGN2011,

May 16-17, 2011

Toulouse, France

${ }^{*}$ Speaker. 


\section{Introduction to polarization}

Polarization, as measured in the radio to optical bands, is an important indicator of the structures of the magnetic fields in synchrotron-emitting jets, and can be measured by VLBI on the sub-pc scales at which much of the $\gamma$-ray and $\mathrm{TeV}$ emission of active galactic nuclei (AGN) is likely to be generated. This brief article reviews the type of information that can be gained by observing jet polarization, with reference to four case studies. Fuller reviews of the physics of AGN jets can be found at $[36,35]$. Polarization monitoring is likely to be very helpful in understanding the physics of AGN in the era of the Cerenkov Telescope Array.

Polarization is usually described by the four Stokes parameters, $(I, Q, U, V) . I$ is the total intensity. $Q$ measures the excess intensity of radiation in the horizontal compared with the vertical direction and $U$ measures the excess in the $+45^{\circ}$ direction relative to the $-45^{\circ}$ direction. $V$ measures the excess intensity of radiation in the right-hand polarization compared with the left-hand polarization. It is common also to use the polarized fraction, $\Pi$, and the position angle of the polarized emission, $\chi$, where

$$
\begin{aligned}
\Pi & =\frac{\left(Q^{2}+U^{2}+V^{2}\right)^{\frac{1}{2}}}{I} \\
\tan 2 \chi & =\frac{U}{Q} .
\end{aligned}
$$

Often it is assumed that $V=0$ and then the linearly polarized fraction, $\Pi_{L}$, is reported rather than the total polarized fraction.

For synchrotron radiation, $I$ measures the product of the energy density in magnetic field and the number density in relativistic electrons and positrons at an energy near $\gamma m_{e} c^{2}$ where the Lorentz factor $\gamma$ is related to the frequency of observation by

$$
\gamma \approx\left(\frac{v}{v_{g}}\right)^{1 / 2}
$$

and $v_{g}$ is the non-relativistic electron gyrofrequency,

$$
v_{g}=\frac{e B}{2 \pi m_{e}}
$$

in SI units. $Q$ and $U$, when compared with $I$, measure the ordering of the magnetic field and indicate its average orientation, again weighted by the number density of relativistic electrons. $V$ is expected to be near zero, unless there are substantial numbers of particles with low Lorentz factor (see §2.3).

If inverse-Compton radiation makes a significant contribution to the jet emission, as it often does in the X-ray band, then the polarization is diluted. Although the inverse-Compton process is intrinsically an anisotropic scattering, in all practical cases the polarization of the inverse-Compton radiation is small. Thus if inverse-Compton and synchtrotron emission occur together, the polarized fraction could be low even if a well-ordered magnetic field is present.

Radio images are often described as showing the orientation of the polarization with "vectors", although the images are always clear in not drawing the polarization as vectors (the lines shown do not have an orientation): polarization is not a vector quantity. It is easy to misinterpret such 
polarization maps - the polarized flux density or polarized fraction maps should not be regarded as tracking field morphology, for example zeros in the polarization do not imply that the field along that line of sight is disordered. The observed polarization direction is also affected by relativistic aberration, and the interpretation of polarization can be complicated in a jet with velocity and electron density substructure.

The usual interpretation of polarization in synchrotron sources is based on the gross morphology of the polarized emission.

- Apparent transverse magnetic fields are usually interpreted in terms of pseudo-random magnetic field that has been compressed by shocks across the jet. Ordered helical or toroidal fields can have similar effects [22].

- Apparent longitudinal magnetic fields are usually interpreted in terms of pseudo-random magnetic field that has been sheared at the edge of a jet, or possibly a large-scale guiding magnetic field, although other possibilities exist, e.g., [25, 37].

- If there is a strong longitudinal field, then a weak shock transverse to the jet can alter $\Pi$ without altering $\chi$, while a strong shock could change $\chi$ by $90^{\circ}$ as well as altering $\Pi$, e.g., [21].

- Oblique shocks can cause changes in $\chi$ by some other angle - the statement that shocks always cause the polarization pattern to align with, or lie transverse to, a jet is incorrect, e.g., $[11,18]$.

- If knots are injected at the base of a jet with oblique field components, then the shears and compressions to which they are subject will cause $\chi$ to rotate with time and position, with some complicated associated changes in $\Pi$.

- A polarized jet knot, moving around a bent jet, can cause the integrated polarization to show a smooth variation in $\chi$ as well as $\Pi$, or a jump in $\chi$ as $\Pi$ passes through a null, e.g., as in 3C 120 [19].

- At radio wavelengths, the observed polarization must be corrected for Faraday rotation effects to infer the value of $\chi$ at emission, and this can be difficult when the jet is embedded in a structured medium with high rotation measure, e.g., [32].

- Most jets show observed $\Pi \ll 70 \%$ (the approximate maximum value from synchrotron emission with a well-ordered field) [30]. This may arise from "beam depolarization", the existence of substructure within the PSF of the observing telescope.

\section{Four case studies}

\subsection{M87}

The Virgo radio galaxy M 87 contains a strong non-thermal jet which displays prominent knots from the radio to X-ray band. The HST-1 knot is particularly interesting because of its recent strong flaring, which led it to remain brighter than the core of M 87 in the X-ray for about three 
years [15]. Cheung et al. [9] found that HST-1 contains sub-components exhibiting superluminal motions (apparent velocities of up to $\sim 4 c$ ) and interpreted HST-1 as a shock in the jet, though the brightest part of HST-1 is only moving at about $0.6 c$ [8]. TeV flares from M 87 reported by [3] have sometimes been associated with HST-1, but it seems clear that the core is the source of the $\mathrm{TeV} \gamma$-rays, especially as the 2008 flare [1,2] was not associated with any strong change at HST-1.

VLBI on M 87 has been a particularly attractive proposition, because, as a very nearby AGN, it is possible to resolve scales of order 100 Schwarzschild radii, and so to image close to the base of the jet collimation zone. The Ly et al. [24] map shows edge-brightening of this inner jet, and detects the counter-jet, leading to a reasonable estimate of the jet speed $(0.5 c)$ and alignment using the usual Doppler preference arguments.

A comparison of the polarization of the inner part of the jet in the radio and the optical [27] shows the polarization to be low in the core in the radio, but relatively high in the optical. There are significant differences in the radio and optical polarization patterns in several of the knots. For most of the jet the polarization is consistent with a relatively well-ordered magnetic field parallel to the jet axis. However, the optical polarization changes significantly at knot brightness maxima and indicates a transverse magnetic field at the upstream ends of knots. This suggests that the opticalemitting electrons are more associated with interior shocks within the jet, while the radio-emitting electrons, with energies $\sim 100$ times lower, are more associated with the sheared region of field near the jet surface. High polarization is also observed between the bright knots, indicating that the field structure in the jet remains well-ordered (mostly in the "shear" sense). A similar interpretation of polarization differences between the radio and optical in terms of a fast spine and a slow sheared sheath has been used to explain recent data for 3C 15 [11], where the polarizations are often not perpendicular to or parallel to the axis of the jet, so that the cylindrical symmetry expected from longitudinal or transverse fields must be broken.

\subsection{CTA 102}

This $z=1.037$ flat-spectrum radio quasar had a relatively quiescent radio light curve from 2009 to early 2011 [31], but just before this meeting it flared strongly as seen by Fermi [10] in a repeat of the flare of 2006 [13]. The structure of the jet in radio total intensity and polarization on milli-arcsec scales has been monitored by VLBI [20,23] and on milli-arcsec scales it displays a fairly typical core-jet morphology, with transverse magnetic fields inferred from the polarization. This "quasar"pattern of field in the inner jet changes to the "BL Lac" pattern, of parallel magnetic field, further out, after a sharp kink in the radio structure.

Superluminal expansion is seen, with speeds up to $15 c$, but with different knots showing different speeds, and the faster motions being seen in both the parallel and transverse magnetic field regions.

\section{$2.33 \mathrm{C} 279$}

This $z=0.536$ flat-spectrum radio quasar is highly variable, and has been studied intensively at milli-arcsecond resolution both to track its rapidly-changing structure and its polarization. VLBA data have shown that $3 \mathrm{C} 279$ displays circular polarization (0.8\%) in the central milliarcsecond of the radio structure [33]. Significant circular polarization has now been detected in more than 10 jets (e.g., [16]), at levels $<1 \%$ in the cores, and up to a few $\%$ at the edges of jets. 
For 3C 279 [34], the surrounding medium can have little effect on the circular polarization, which therefore was attributed to

- the synchrotron emission itself, which requires a highly ordered magnetic field, and an electron-proton plasma, and/or

- internal conversion from linear polarization, via changing perpendicular magnetic fields, or internal Faraday rotation.

The sign of the polarization changes at $7 \mathrm{~mm}$ [33] suggesting that the circular polarization arises through Faraday conversion in the optically-thick parts of the source: an inhomogeneous core, or in a structured inner jet. Since the polarization tends to increase with increasing frequency [33] infer that these inner source regions show significant inhomogeneity. Plausible Faraday conversion models put fewer constraints on $\gamma_{\min }$ than was first thought [17].

\section{$2.43 C 346$}

A rather different example of polarization effects is displayed by the $z=0.162$ radio galaxy 3C 346 [12]. The apparent magnetic field direction in the jet, as inferred from radio and optical polarization, shows a pronounced change at a sharp $\left(70^{\circ}\right)$ kink at knot $\mathrm{C}$. This can be interpreted as an oblique shock, where the underlying flow turns at a plane and the compression associated with the shock changes the alignment of the mean field. A detailed model of the polarization structure shows that this explanation requires moderate projection effects (so that the true kink angle in the jet is about one third the apparent angle), and a jet speed that remains relativistic $(\sim 0.9 c)$ to distances of several kpc from the nucleus of the galaxy.

\section{Generalizations}

Low-power jets show synchrotron spectra from the radio to the X-ray, with a spectral break in the infra-red or optical. This break energy corresponds to typical electron energies of order $1 \mathrm{TeV}$, and the amplitude of the spectral break exceeds the canonical break of 0.5 . This is likely to arise from the balance between electron acceleration and energy losses, in the presence of electron diffusion and the bulk dynamics of the flow. Similar spectra are seen in knots and diffuse parts of the jets, but the interpretation is complicated by the offsets between the radio and X-ray knot locations, which are frequently measured to be far larger than the few pc that the X-ray emitting electrons can travel in an energy loss time. On VLBI scales the structures of the inner jets tend to be one-sided (as in the higher-power sources), but two-sided VLBI jets are seen in a large minority of both FR I (jetty) and FR II (hot-spot) radio sources, and most jets tend to be subluminal in most regions. The VLBI structures are confused by high free-free optical depths in some objects (e.g., NGC 4261 [28]), but optical depth data may allow mapping of the outer accretion torus in an AGN. If much external material is present, then the structures of the polarizations will be confused by Faraday rotation (undoubtedly time-variable, because of changes in the medium and jet motions). The polarization in such jets tends to follow the shear/shock model, rather like M 87, and polarizations of up to about $50 \%$ are common, with the apparent magnetic field usually being longitudinal. 
In high-power jets (BL Lacs or flat-spectrum radio quasars), the overall spectrum of the inner jets plus cores tends to show the synchrotron self-Compton "second peak" in the X-ray or $\gamma$-ray, where the bulk of the emission likely comes from the most compact regions, near the AGN. On kpc scales, the jets can have $\mathrm{X}$-ray spectra that are as flat as the radio spectra, indicating their origin also in inverse-Compton emission - but here from the scattering of an external radiation field by the synchtron-emitting electrons. In either the inner or outer parts of the source, relativistic boosting of the jets is essential for the inverse-Compton emission to reach the brightness that is seen. In BL Lac objects it is common for the kpc-scale jets to have rather steep X-ray spectra, indicating a synchrotron rather than inverse-Compton origin (e.g., in PKS 0521-365 [6]). Generally, the polarization in BL Lac objects is characterized by low rotation measures, and the inferred magnetic field is transverse in a small majority $\left(\frac{2}{3}\right)$ of the jets [14]. Some BL Lac jets show longitudinal fields at their edges and transverse fields at their centres, resembling the shear/shock model of lowpower kpc-scale radio jets. At cm wavelengths, the fractional polarization $\Pi \sim 5 \%$ in much of the emission, but in knots $\Pi$ can rise to the theoretical maximum of about $70 \%$.

Quasars typically show lower polarizations than BL Lac objects $(<2 \%)$. Knot speeds tend to be higher than in BL Lacs, up to tens of $c$, with samples of quasars showing a wide spread in apparent velocities. The jet polarizations usually imply parallel ordering in the magnetic fields, though transverse fields are certainly seen (epecially in knots). On kpc scales the magnetic fields tend to be lower than in low-power objects, and jets show extreme bends, presumably from projection effects. The interpretation of the radio polarization on VLBI (and larger) scales can be strongly affected by surrounding plasma. Taylor [32] found that some jets display rotation measures as large as $2000 \mathrm{radm}^{-2}$, although the typical values are less than $100 \mathrm{radm}^{-2}$. The high rotation measures show that dense magnetized plasma is present out to about $30 \mathrm{pc}$ from the nuclei of $3 \mathrm{C} 273$, 3C 279, and 3C 380. High rotation measures are, in some cases, associated with jet structures, such as deflections (e.g., in 3C 119 [26]), and high rotation measure gradients may cause significant depolarization in VLBI data (e.g., in 3C 273 [5]). The polarization can also be interpreted in terms of inner spine-sheath structures (e.g., in PKS 1055+01 [4]). In some cases very fast polarization changes are detected, with the polarization changing more rapidly than the intensity. While some of these changes are likely to be propagation effects, some may be intrinsic (e.g., in 0954+658), and in the future it will be very interesting to see whether polarization effects associated with shocks in the inner jets can be associated with $\mathrm{TeV}$ flares.

\section{Summary}

- Fermi and VHE $\gamma$-ray AGN display pc-scale relativistic jets, and the high-energy activity of such AGN is associated with detectable intensity and polarization changes in the jets caused by the variable flow.

- VLBI monitors speeds of jet flows via the structure changes, and can also follow changes in the configuration of the magnetic field from polarization. Large and rapid polarization changes are expected from the twin influences of shear and shocks.

- VLBI data are looking at structure beyond the scale of the $\gamma$-ray emitting zone in most cases, because the base of the jet is optically thick at most radio wavelengths, but mm-wave VLBI 
is now getting close to the scale of the collimation region in the closest AGN.

- Simultaneous high-sensitivity $\gamma$-ray and mm-wave VLBI observations of the nearest AGN should be planned routinely in the CTA era, to relate $\gamma$-ray flares to the changing state of the jet flows and to see the extent of the flow changes that can occur without high-energy radiation being generated.

\section{References}

[1] A.A. Abdo et al. 2009. Fermi Large Area Telescope gamma-ray detection of the radio galaxy M 87, ApJ 707, 55.

[2] Acciari et al. 2009. Radio imaging of the very-high energy $\gamma$-ray emission region in the central engine of a radio galaxy, Sci 325, 444.

[3] F. Aharonian et al. 2006. Fast variability of tera-electron volt $\gamma$-rays from the radio galaxy M 87, Sci 314, 1424.

[4] J.M. Attridge, D.H. Roberts, J.F.C. Wardle, 1999, Radio jet-ambient medium interactions on parsec scales in the blazar $1055+018$, ApJ 518, L87.

[5] J.M. Attridge, J.F.C. Wardle, D.C. Homan, 2005, Concurrent 43 and $86 \mathrm{GHz}$ very long baseline polarimetry of $3 C 273$, ApJ 633, L85.

[6] M. Birkinshaw, D.M. Worrall, M.J. Hardcastle, 2002, The X-ray jet and halo of PKS0521-365, MNRAS 335, 142.

[7] S. Britzen et al. 1999. The CJF survey - first results on superluminal motion, ASPC 159, 431.

[8] C.S. Chang, E. Ros, Y.Y. Kovalev, M.L. Lister, 2010. VLBI detection of the HST-1 feature in the M 87 jet at $2 \mathrm{~cm}, A \& A \mathbf{5 1 5}, 38$.

[9] C.C. Cheung, D.E. Harris, L. Stawarz, 2007. Superluminal radio features in the M 87 jet and the site of flaring TeV gamma-ray emission, ApJ 663, L65.

[10] S. Ciprini, 2011. Fermi detection of a GeV flare from blazar CTA 102, ATel\#3320.

[11] F. Dulwich et al., 2007, The structure of the jet in 3C 15 from multi-band polarimetry, MNRAS 374, 1216.

[12] F. Dulwich et al., 2009, The magnetic field and geometry of the oblique shock in the jet of 3C 346, MNRAS 398, 1207.

[13] C.M. Fromm et al., 2010, Shock-shock interaction in the jet of CTA 102, arXiv:1011.4825.

[14] D.C. Gabuzda, A.B. Pushkarev, T.V. Cawthorne, 2000, Analysis of $\lambda=6 \mathrm{~cm}$ VLBI polarization observations of a complete sample of southern BL Lacertae objects, MNRAS 319, 1109.

[15] D.E. Harris et al., 2006, The outburst of HST-1 in the M 87 jet, ApJ 640, 211.

[16] D.C. Homan, M.L. Lister, 2006, MOJAVE: monitoring of jets in active galactic nuclei with VLBA experiments. II. First-epoch $15 \mathrm{GHz}$ circular polarization results, AJ 131, 1262.

[17] D.C. Homan et al., 2009, Full polarization spectra of 3C 279, ApJ 696, 328.

[18] P.A. Hughes, M.F. Aller, H.D. Aller, 2011, Oblique shocks as the origin of radio to gamma-ray variability in active galactic nuclei, ApJ 735, 81. 
[19] J.L. Gómez, A.P. Marscher, A. Alberdi, S.G. Jorstad, I. Agudo, 2001, Monthly 43 GHz VLBA polarimetric monitoring of $3 C 120$ over 16 epochs: evidence for trailing shocks in a relativistic jet, ApJ 561, L161.

[20] S.G. Jorstad et al. 2005. Polarimetric observations of 15 active galactic nuclei at high frequencies: jet kinematics from bimonthly monitoring with the Very Long Baseline Array, AJ 130, 1418.

[21] S.G. Jorstad et al. 2007. Multiwaveband polarimetric observations of 15 active galatic nuclei at high frequencies: correlated polarization behaviour, AJ 134, 799.

[22] R.A. Laing 1981. Magnetic fields in extragalactic radio sources, ApJ 248, 87.

[23] M.L. Lister et al. 2009, MOJAVE: monitoring of jets in active galactic nuclei with VLBA experiments: V. Multi-epoch VLBA images, AJ 137, 3718.

[24] C. Ly, R.C. Walker, W. Junor, 2007, High frequency VLBI imaging of the jet base of M 87, ApJ 660, 200.

[25] M. Lyutikov, V.I. Pariev, D.C. Gabuzda, 2005. Polarization and structure of relativistic parsec-scale AGN jets, MNRAS 360, 869.

[26] R. Nan et al. 1999. VLBI polarimetry of the high rotation measure source 3C 119 at $8.4 \mathrm{GHz}, \mathrm{A \& A}$ 344, 402.

[27] E.S. Perlman et al. 1999. Optical and radio polarimetry of the M 87 jet at $0.2^{\prime \prime}$ resolution, AJ 117, 2185.

[28] B.G. Piner, D.L. Jones, A.E. Wehrle, 2001, Orientation and speed of the parsec-scale jet in NGC 4261 (3C 270), AJ 122, 2954.

[29] B.G. Piner, N. Pant, P.G. Edwards, 2010, The jets of TeV blazars at higher resolution: 43 GHz and polarimetric VLBA observations from 2005 to 2009, ApJ 723, 1150.

[30] L.K. Pollack, G.B. Taylor, R.T. Zavala, 2003. VLBI polarimetry of 177 sources from the Caltech-Jodrell Bank flat-spectrum survey, ApJ 589, 733.

[31] J.L. Richards et al., 2011, Blazars in the Fermi era: the OVRO 40m telescope monitoring program, ApJS 194, 29.

[32] G.B. Taylor, 1998, Magnetic fields in quasar cores, ApJ 506, 637.

[33] V.M. Vitrishchak et al., 2008, The 15-43 GHz parsec-scale circular polarization of 41 active galactic nuclei, MNRAS 391, 124.

[34] J.F.C. Wardle, D.C. Homan, R. Ojha, D.H. Roberts, 1998, Electron-positron jets associated with the quasar 3C 279, Nat 395, 457.

[35] D.M. Worrall, 2009, The X-ray jets of active galactic nuclei, A\&A Rev 17, 1.

[36] D.M. Worrall, M. Birkinshaw, 2006, Multiwavelength evidence of the physical processes in radio jets, in Physics of active galactic nuclei at all scales, eds. D. Alloin, R. Johnson, P. Lira; Springer, Berlin.

[37] N.L. Zakamska, M.C. Begelman, R.D. Blandford, 2008. Hot self-similar relativistic magnetohydrodynamic flows, ApJ 679, 990. 